\title{
计算机应用的现状与计算机的发展趋势
}

季江

江苏龙典建设集团有限公司

DOI: 10.18686/bd.v1i11.1082

[摘要] 随着社会的不断发展,科技的不断进步, 我国各个领域近年来都得到了很大的发展, 尤其是在计算机被广泛应用之 后, 各个领域的相关技术得到了改善,人们的生活水平也得到了显著提高,所带来的经济效益也呈不断上升的趋势, 经过多年 发展如今计算机在人们的生活和工作中以及成为不可或缺的一部分。

[关键词] 计算机; 应用现状; 发展趋势

随着计算机用户的不断增加, 计算机以其独特的生命 力感染着社会变革的进程。我国居民家庭计算机拥有率从 二十世纪末的百分之十跃升到当今的百分之八十以上,此 外,企事业单位、教育机构等组织也开始了计算机的应用和 普及,网络化和信息化已经成为我国经济增长的必要工具。 从国际科学水平来看, 计算机的技术含量已经成为评价国 家综合实力的基础, 也是实现现代化社会发展的必经之 路。

\section{1 计算机应用技术的概述}

所谓的计算机应用技术就是指研究计算机应用于社会 中各个行业和领域的理论、技术、方法以及系统的一门边缘 性的学科,它计算机学生的组成的很重要的一部分, 它也是 促进计算机学科与其他学科有效融合的一个载体。通常情 况下, 计算机应用的分类一般分为数值计算领域和非数值 应用领域两大类, 这两大领域都具备着自身独特的特点,但 对于促进科学技术的进步都是有着重要的作用的。

\section{2 计算机发展历程}

从我国应用计算机的历史长河中来看, 我国首次出现 对计算机技术的使用在上个世纪 40 年代,这一阶段中无论 是经济实力还是科研力量都不能够支持计算机多方面的应 用,只能将计算机数据方面应用在军事领域。50 年代的计算 机探讨从数值方面转向非数值方面, 应用领域也由军事领 域想工商业、企业方面延伸。真正实现计算机技术各方面的 广泛应用是在 70 年代,这一时期的科学技术水平发展迅速, 为计算机技术研发提供有效的后盾支持, 计算机各项研发 成果被广泛的应用在服务行业、农业及文化教育中。同时， 网络技术的发展大大推动了计算机的应用, 人类社会开始 迎来信息化时代。

\section{3 我国计算机发展现状}

3.1 可喜之处

随着我国对计算机重视程度的不断增加以及经济水 平、人民生活水平的不断提升,计算机在我国的各个领域发 挥着重要的作用。从农业上讲,农业生产流通信息网络的建 立和完善使得农民能够更加具体、更加准确的了解到市场 上的供求信息, 从而根据互联网所提供的数据进行进一步
的生产作业计划。从影视娱乐创作来讲,我们所熟知的影视 创作例如电影、电视剧、动画等均离不开互联网和计算机的 应用,数字音乐也是近几年我们所关注的重要内容,数字音 乐从制作到流通均离不开计算机的应用。从教育领域来看, 近几年来,随着网络教学的兴起,计算机在我们的日常教学 中发挥着越来越重要的作用, 网上授课使教学打破了时间 和地点的限制, 同时使得教育资源得到了进一步的有效利 用。从人民的日常生活来看,计算机不仅仅丰富了民众的日 常生活,使得教育资源得到了进一步的有效利用,计算机在 公安领域的应用同样进一步的保障了居民的安全, 其中最 值得一提的是计算机在商业领域的应用, 近几年来网络购 物在我们的日常生活中发挥着越来越重要的作用, 也逐渐 成为了我们日常生活中不可或缺的服务之一, 这一领域的 发展使得计算机在我们的生活中也发挥着越来越重要的作 用。

\section{2 可忧之处}

我国计算机领域仍然存在着一定的不足和改进的空 间,主要体现在以下的几个方面。第一,信息化水平较低,我 国现有的信息化水平不能够更好的满足我国计算机的发展 水平人们的需求, 同时我国大型工程的软硬件产品大多来 源于国外,依赖于国外进口, 这就使得我国相关的技术领域 对国外产品有着较大的依赖性,丧失了自主创新的能力。第 二, 相关的法律法规有待进一步的发展, 随着互联网以及计 算机应用范围的不断扩大, 我国计算机领域也不可避免的 出现了一系列的问题, 针对这一问题提出合理的改进措施 并进一步完善相关的法律法规是我们在规范计算机市场过 程中的主要任务。

\section{4 计算机在社会发展中的作用}

计算机在社会发展中的作用主要体现在：促进社会发 展信息化和增加社会的交往两个方面。随着计算机在我国 生产工作中的应用, 计算机的一系列功能支撑着各项工作 平稳有序的进行。计算机将更多的数据信息存储在电脑中, 实现资源共享,为企业信息化发展提供基本的条件;计算机 应用极大地改善了人类生活原有的交流方式, 传统社会发 展中,人们靠书信进行沟通,其传递时间严重影响了信息及 


\section{关于绿色建筑和建筑节能设计的探讨}

郝兵

齐齐哈尔市房屋征收办公室

DOI:10.18686/bd.v1i11.1090

[摘 要] 绿色建筑是指在建筑的全寿命周期内,最大限度地节约资源(节能、节地、节水、节材)、保护环境和减少污染, 为人 们提供健康、适用和高效的使用空间,与自然和谐共生的建筑。

[关键词] 绿色建筑; 建筑节能; 节能; 设计

我国的经济和社会正保持着高速发展的态势, 处于工 业化和城镇化快速发展阶段,工业的增长、居民消费结构的 升级,特别是城镇化进程的快速发展, 对能源、经济资源的需 求将更加迫切。这样,我们不得不正视我国能源(特别是不可 再生能源)、土地、水、原材料等资源严重短缺而实际利用效

时的传递,甚至会造成信息的丢失,而信息化的交流方式,改 变书信传递消息的弊端,为人们的交流。

\section{5 计算机发展趋势的预测}

计算机的进一步发展主要依赖于计算机技术的进一步 发展,随着我国市场需求的不断提升,对计算机技术水平的 要求也不断提升, 在这样的情况下进一步发挥人才的优势, 提高自主创新能力, 在现有技术的基础上进行进一步的创 新,从而促进新兴技术的进一步应用。

5.1 光计算机

光计算机是计算机未来发展的主要趋势, 其具有以下 几个优点:

首先, 光器件允许高光频率的大范围通过, 也就意味着 具有很大的库存带宽以及较大的传输处理的信息量。如果 出现两束出现干涉的光, 两束光之间就必须要具有相同的 频率以及相同的初始位相差和振动方向。所以,大量的波长 不同、波长相同振动方向不同的光波可以同时在同一根光 导纤维中行进,而且它们当中不会出现相互干涉的情况。

其次, 光计算机信息传输的过程中具有很小的会发生 畸变和失真的概率, 而且具有很快的运算速度。在介质中光 与电都有极快的传播速度,但两者还是存在一些差异。光计 算机就是所谓的 “ $F$ 我” 导线计算机,在光介质中光可以实 现无寄生电感、电容、电阻等问题的快速传播,并且没有接 地电位差。这就使信息畸变和信息失真在传输过程中大大 降低, 相对于电子器件而言, 光器件的开关速度非常之快。

5.2 量子计算机

量子计算机在平行运算处理方面具有很大的优势。例 如,将一份遗失文件从一个非常大的办公楼当中找出来。要 是对传统计算机的寻找方式予以利用就必须对每个办公室 进行逐一查询, 而量子计算机就可以以寻找者提出的条件 和要求为根据, 方便快捷的将与被寻找文件最为相似的副
率低、环境污染严重且仍在不断加剧的事实。因此中国要走 可持续发展道路,发展绿色建筑与节能建筑是刻不容缓的。

\section{1 建筑能耗的基本内容}

1.1 什么是建筑能耗

建筑能耗的定义: 民用建筑在运行中所产生的能耗, 即
本复制出来,同时进人所有办公室同时搜索。

\section{3 化学计算机}

化学计算机的运行机理是以一种名为微观碳分子的化 学制品为信息载体,以实现信息的储存于传输。因此,其体积 更小、运算速度更快、计算机能力更大,在信息传输速度的 方面甚至可能超过人脑, 因此发展前景巨大。如何取代硅电 子部件来研究碳基制品是化学计算机发展的关键所在。虽 然这一领域现今还处于探索阶段, 但也已取得了不少进展, 前景可观。

\section{4 生物计算机}

所谓的生物计算机是指具有生物活性, 能够与人体组 织进行结合的计算机, 其尤其可以与人类的大脑和神经系 统进行联系。如此以来,大脑就可以直接指挥生物计算机,使 其成为大脑的一个辅助或扩充装置, 并由人体的细胞来为 其补充能量,无需外界的能源补充。生物计算机将成为人类 学习、思考和创造的帮手,并且能耗极小。

\section{6 结束语}

综上所述, 计算机如今已经完全融人到人们的日常生 活和工作中,并且正在发挥着越来越重要的作用,并且科学 技术的不断更新和完善, 也会使得计算机技术持续发展, 如 今经济全球化的趋势也越来越明显, 我国各个领域也在不 断加快与国际接轨的步伐。

\section{参考文献:}

[1] 梁文宇. 计算机应用的现状与计算机的发展趋势 [J].科技经济市场,2017,(02):188-190.

[2] 刘青梅. 计算机应用的现状与计算机的发展趋势 [J].电脑知识与技术, 2016,12(25): 193-194.

[3]李洋.计算机应用的发展现状和发展趋势[J].科技 传播,2016,8(17):107-108. 